\title{
Association between phthalates and attention deficit disorder and learning disability in U.S. children, $6-15$ years
}

\author{
Vidita Chopra ${ }^{a}$, Kim Harley ${ }^{\mathrm{a}}$, Maureen Lahiff ${ }^{\mathrm{b}}$, Brenda Eskenazi ${ }^{\mathrm{a}, *}$ \\ ${ }^{a}$ Center for Environmental Research and Children's Health (CERCH), School of Public Health, University of California Berkeley, Berkeley, CA 94720, USA \\ ${ }^{\mathrm{b}}$ Division of Biostatistics, School of Public Health, University of California Berkeley, Berkeley, CA, USA
}

\section{A R T I C L E I N F O}

\section{Article history:}

Received 30 May 2013

Received in revised form

29 October 2013

Accepted 31 October 2013

Available online 19 November 2013

\section{Keywords}

Attention deficit disorder

Learning disability

Phthalates

Plasticizers

National Health and Nutrition

Examination Survey

\begin{abstract}
A B S T R A C T
Objective: This study investigates the association between urinary phthalate metabolite levels and attention deficit disorder (ADD), learning disability (LD), and co-occurrence of ADD and LD in 6-15-yearold children.

Methods: We used cross-sectional data from the National Health and Nutrition Examination Survey (NHANES, 2001-2004). Phthalate metabolites with $\geq 75 \%$ detection in urine samples were examined. The study population comprised 1493 children with parent-reported information on ADD or LD diagnosis and phthalate concentrations in urine. Phthalate concentrations were creatinine-adjusted and $\log _{10}$ transformed for analysis. All models controlled for child sex, age, race, household income, blood lead, and maternal smoking during pregnancy.

Results: There were 112 ADD cases, 173 LD cases, and 56 ADD and LD cases in the sample. After adjusting for potential confounders, we found increased odds of ADD with increasing urinary concentration of di2-ethylhexyl phthalates (OR: 2.1; 95\% CI: 1.1, 3.9) and high molecular weight phthalates (OR: 2.7; 95\% CI: 1.2, 6.1). In addition, dibutyl phthalates (OR: 3.3; 95\% CI: 0.9, 12.7) and high molecular weight phthalates (OR: 3.7; 95\% CI: 0.9, 14.8) were marginally associated with increased odds of co-occurring ADD and LD. We did not find associations for any phthalate and LD alone. We observed stronger associations between phthalates and ADD and both ADD and LD in girls than boys in some models.

Conclusions: We found cross-sectional evidence that certain phthalates are associated with increased odds of ADD and both ADD and LD. Further investigations with longitudinal data are needed to confirm these results.
\end{abstract}

(c) 2013 Elsevier Inc. All rights reserved.

\section{Introduction}

Phthalates are industrial chemicals often used in personal care products and to soften plastics in toys, household items such as food containers, and medical devices (Barr et al., 2003; Hauser and Calafat, 2005; Sathyanarayana et al., 2008). Because they are not chemically bound to the plastics, they disperse into the environment easily, leading to exposure via inhalation, ingestion, and dermal contact (Afshari et al., 2004; Clark et al., 2003). In the

\footnotetext{
Abbreviations: High M.W, high molecular weight; Low M.W, low molecular weight; DEHP, di-2-ethylhexyl phthalate; DBP, dibutyl phthalate; MBzP, monobenzyl phthalate; MEP, mono-ethyl phthalate; MCPP, mono-(3-carboxypropyl) phthalate; ADHD, attention-deficit/hyperactivity disorder; ADD, attention deficit disorder; LD, learning disability; OR, odds ratio; CI, confidence interval; NHANES, National Health and Nutrition Examination Survey; CDC, Centers for Disease Control and Prevention; DSM-IV, Diagnostic and Statistical Manual of Mental Disorders: Fourth Edition; PIR, poverty income ratio; LOD, limit of detection

* Corresponding author. Fax: +1 5106429083.

E-mail addresses: choprav@obgyn.ucsf.edu (V. Chopra), eskenazi@berkeley.edu (B. Eskenazi).
}

National Health and Nutrition Examination Survey (NHANES) 1999-2000, urinary metabolites of phthalates were detectable in $75-90 \%$ of the samples, indicating widespread exposure in the U.S. population (Silva et al., 2004).

Eight different phthalate diesters are in general consumer use. High molecular weight (high M.W.) phthalates, including benzylbutyl phthalate (BzBP), di-2-ethylhexyl phthalate (DEHP), di-isononyl phthalate (DiNP), dimethyl phthalate (DMP), and di-noctyl phthalate (DOP), are commonly found in vinyl tubing, flooring, and wall covering (NRC, 2008). DEHP, the most commonly used phthalate ester, is the dominant plasticizer in polyvinyl chloride (PVC) due to its low cost (Afshari et al., 2004; Clausen et al., 2004). Low molecular weight (low M.W.) phthalates, such as dibutyl phthalates (DBP), dicylohexyl phthalate (DCHP), diethyl phthalate (DEP), are found in adhesives, printing ink, personal care products, and nail polish (NRC, 2008).

In animal studies, phthalates have been demonstrated to be endocrine disruptors that exhibit weak estrogenic activity, impair thyroid function, and affect neurodevelopment (Boas et al., 2010; Colborn, 2004; Crofton, 2008; Kavlock et al., 1996). In rats, prenatal 
and neonatal exposure to DEHP or DBP results in impaired neuronal growth and activity (Arcadi et al., 1998; Chen et al., 2011; Li et al., 2009; Tanaka, 2005; Tanida et al., 2009). Neonatal dosing of rats with $87 \mathrm{nmol}(29 \mu \mathrm{g})$ of DCHP, DBP and DEHP via intracisternal injection was associated with increased motor hyperactivity at 4-5 weeks of age (Ishido et al., 2004; Masuo et al., 2004). Exposure to higher levels $(10 \mathrm{mg} / \mathrm{kg}$ ) of DEHP during the third week after birth was associated with reduced hippocampal development in male, but not female, rats (Smith et al., 2011).

A small number of studies have assessed the association of prenatal or postnatal phthalate exposure and neurobehavioral development in humans (Engel et al., 2010, 2009; Kim et al., 2009; Whyatt et al., 2012). In a study from New York City (NYC), higher concentrations of prenatal urinary metabolites of low and high M.W. phthalates were linked to decrements in motor and mental development and increased odds of internalizing behavior problems in 3-year-olds $(N=319)$ (Whyatt et al., 2012). In another birth cohort from NYC $(N=188)$, prenatal urinary levels of low M.W. phthalates were associated with increased odds of externalizing behaviors such as aggression, conduct problems, and attention problems in 4-9-year-old children (Engel et al., 2010). Postnatal exposure to phthalates has been assessed in one previous study; child urinary concentrations of DEHP metabolites were found to be related to attention-deficit/hyperactivity disorder (ADHD) in 8-11year-old Korean children $(N=261)$ (Kim et al., 2009). Thus, there is growing evidence that phthalate exposure may be related to attention and behavioral problems.

The Diagnostic and Statistical Manual of Mental Disorders, Fourth Edition (DSM-IV) categorizes ADD into three subtypes: predominantly inattentive, predominantly hyperactive/impulsive, or combined (APA, 1994). ADD and learning disability (LD) are the most frequent neurobehavioral disorders diagnosed in children. These disorders affect $5-12 \%$ of school-age children in the United States (Vakil et al., 2012). The co-occurrence of ADD and LD is of concern due to its association with worse executive functioning in children than either alone (Mattison and Mayes, 2012). This study investigates the association between urinary phthalate metabolite levels and ADD, LD, and the co-occurrence of ADD and LD in 6-15-yearold children in NHANES (2001-2004).

\section{Methods}

\subsection{Study design and participants}

The study sample was 6-15-year-old children who participated in the 20012004 cycles of the National Health and Nutrition Examination Survey (NHANES), a population-based, annual health survey of the United States (U.S.). NHANES data were collected using a complex, multistage, probability sampling design of the civilian non-institutionalized U.S. population, and certain subgroups were oversampled. Details of the NHANES study have been published elsewhere (CDC, 2012). Participant's parents or guardians completed home interviews about their demographic, socioeconomic, and health information. Urine samples were collected during physical examinations in mobile units (NHANES, 2004).

NHANES data from the 2001-2002 and 2003-2004 cycles were combined, as these cycles provided both information on parent/guardian report of ADD and LD and measures of urinary phthalate metabolite concentrations in a subsample of 1494 participants aged 6-15 years. We excluded individuals missing $\operatorname{ADD}(N=3)$ or LD $(N=1)$ data, for final sample sizes of 1491 for ADD analyses and 1493 for LD analyses. For analyses of individuals diagnosed with co-occurrence of ADD and LD, the comparison group were those who were diagnosed with neither; therefore, we excluded 172 individuals who had answered "yes" to having only ADD or LD, for a total of $N=1318$ for these analyses.

\section{2. $A D D$ and/or $L D$ diagnoses}

Information about the child's ADD or LD diagnosis was based on the parent or guardian's response to the questions "Has a doctor or health professional ever told you that your child had attention deficit disorder?" and "Has a representative from a school or a health professional ever told you that your child had a learning disability?" In our study population of 6-15-year-olds, there were 112 ADD cases,
173 LD cases, and 56 cases with co-occurring ADD and LD. The 56 participants with ADD and LD were a subset of those who had ADD or LD.

\subsection{Urinary phthalate metabolite measurements}

After collection, urine samples were stored at $-20^{\circ} \mathrm{C}$ until shipment to the centers for Disease Control and Prevention (CDC) for testing. Details on the laboratory procedures have been published elsewhere (CDC, 2006). Briefly, high performance liquid chromatography-electrospray ionization-tandem mass spectrometry (HPLC-ESI-MS/MS) was used to detect concentrations of phthalate metabolites in urine. The samples were processed by the enzymatic deconjugation of the glucuronidated phthalate monoesters, which was followed by solid phase extraction (NHANES, 2004). Concentrations below the limit of detection (LOD) were assigned the value of the detection limit divided by $\sqrt{ } 2$ (Hornung and Reed, 1990). Eight phthalate groups were measured in the 2001-2004 NHANES data sets.

We limited our analyses on those phthalates whose metabolites were detected in $\geq 75 \%$ of samples, namely sum $(\Sigma)$ DEHP, $\Sigma$ DBP, BzBP, DEP, and DOP. DEHP exposure was estimated by summing the values of three DEHP metabolites, mono(2-ethyl)-hexyl phthalate (MEHP), mono- (2-ethyl-5-hydroxyhexyl) phthalate (MEHHP), and mono- (2-ethyl-5-oxohexyl) phthalate (MEOHP). DBP devolves to two metabolites, mono-n-butyl phthalate (MnBP) and mono-isobutyl phthalate (MiBP), which were summed to obtain the $\Sigma$ DBP variable. Three phthalates were estimated using a single metabolite: DOP devolves to mono- (3-carboxypropyl) phthalate (MCPP), BzBP to mono-benzyl phthalate (MBzP), and DEP to mono-ethyl phthalate (MEP) (CDC, 2009).

Additionally, we created variables for the sum of the high M.W. ( $>250 \mathrm{Da}$ ) and low M.W. ( $<250 \mathrm{Da})$ phthalates, as they come from similar sources and have similar biological activity (Engel et al., 2010; NRC, 2008; Wolff et al., 2008). The $\Sigma$ high M.W. variable comprised MBzP+MCPP + MEHP + MEHHP + MEOHP, the $\Sigma l o w$ M.W. variable was MEP+MMP+MiBP + MnBP.

\subsection{Statistical analysis}

All statistical analyses were performed using STATA/IC (version 11.1; StataCorp LP, College Station, TX, USA). Since NHANES is a multi-stage probability design, appropriate weights, primary sampling units, and strata were applied to the data to obtain robust standard errors and unbiased point estimates (CDC, 2011).

Potential confounders considered a priori were: age, sex, race, household income (poverty-income ratio (PIR)), low birth weight, health insurance coverage, routine source of healthcare, mental health professional use in past year, child blood lead concentration ( $\log _{10}$ transformed), maternal age at birth, and maternal smoking during pregnancy. Variables were kept in the models if they were associated with any of the exposure and outcome variables at $p<0.10$ or if their exclusion changed the main effect by $>10 \%$. Covariates included in the final models were: child sex (M/F), age (continuous), race (White/Black/Mexican American/Other Hispanic/Other), household income ( $<1 \mathrm{PIR} / 1-2.99 \mathrm{PIR} / 3-4.99 \mathrm{PIR} / \geq 5$ $\mathrm{PIR})$, blood lead (continuous), and maternal smoking during pregnancy $(\mathrm{Y} / \mathrm{N})$.

All phthalate concentrations were adjusted for creatinine to account for urine dilution (Jackson, 1966), summed, and $\log _{10}$ transformed to approximate a normal distribution and minimize the effects of outliers. In sensitivity analyses, we examined non-creatinine-adjusted phthalate concentrations or included $\log _{10}$ transformed urinary creatinine in the model (Silva et al., 2004).

Logistic regression was used to estimate odds ratios (ORs) and 95\% confidence intervals (CIs) for ADD, LD, and both ADD and LD, per 10-fold increase in $\Sigma$ DEHP, $\Sigma$ DBP, MBzP, MEP, MCPP, $\Sigma$ high M.W. and $\Sigma$ low M.W. phthalate concentrations ( $\mu \mathrm{g} / \mathrm{g}$ creatinine). Statistical interactions of phthalate variables with lead and sex were investigated in the multivariate models using cross-product terms. Interaction terms with $p<0.10$ in the model were considered statistically significant.

\section{Results}

The weighted prevalence of ADD was $9.1 \%$, LD was $12.1 \%$, and co-occurrence of ADD and LD was 5.9\%. The demographic characteristics of the study population are listed in Table 1. Most participants had health insurance $(87 \%)$ and a routine source of medical care (94\%). The mean child age was 10.5 years $(95 \% \mathrm{CI}$ : $10.3,10.8)$ and mean child blood lead levels were $1.3 \mu \mathrm{g} / \mathrm{dL}(95 \% \mathrm{CI}$ : $1.2,1.4)$. Metabolites of $\Sigma$ DEHP, $\Sigma$ DBP, MBzP, MEP and MCPP were detected in almost all individuals, with the highest concentrations seen for MEP (Table 2). When comparing geometric mean (GM) phthalate metabolite concentrations by diagnosis of ADD only, LD only, both ADD and LD, or neither, significant differences were seen for $\Sigma$ DEHP and $\Sigma$ high M.W. phthalates, with the highest concentrations in those with both ADD and LD (Table 3). 
Table 1

Demographic characteristics of 6-15 year old children in our study population, NHANES (2001-2004).

\begin{tabular}{|c|c|c|}
\hline Population characteristics & $N^{\mathrm{a}}$ & Weighted (\%) \\
\hline \multicolumn{3}{|l|}{$\operatorname{Sex}(N=1493)$} \\
\hline Male & 726 & 51.5 \\
\hline Female & 767 & 48.5 \\
\hline \multicolumn{3}{|l|}{ Race $(N=1493)$} \\
\hline White & 399 & 61.1 \\
\hline Black & 532 & 15.5 \\
\hline Mexican American & 461 & 11.5 \\
\hline Other hispanic & 50 & 6.5 \\
\hline Other & 51 & 5.4 \\
\hline \multicolumn{3}{|l|}{ Household income $(N=1428)$} \\
\hline$<1.00 \mathrm{PIR}^{\mathrm{b}}$ & 473 & 23.6 \\
\hline 1.00-2.99 PIR & 585 & 38.7 \\
\hline 3.00-4.99 PIR & 239 & 23.0 \\
\hline$\geq 5.00$ PIR & 131 & 14.7 \\
\hline \multicolumn{3}{|c|}{ Low birth weight $(<2500 \mathrm{~g})(N=1422)$} \\
\hline Yes & 196 & 13.2 \\
\hline No & 1226 & 86.8 \\
\hline \multicolumn{3}{|c|}{ Health insurance coverage $(N=1483)$} \\
\hline Yes & 1244 & 87.2 \\
\hline No & 239 & 12.8 \\
\hline \multicolumn{3}{|c|}{ Routine place for healthcare $(N=1493)$} \\
\hline Yes & 1370 & 93.9 \\
\hline No & 123 & 6.1 \\
\hline \multicolumn{3}{|c|}{ Seen mental health professional in past year $(N=1493)$} \\
\hline Yes & 121 & 9.8 \\
\hline No & 1372 & 90.2 \\
\hline \multicolumn{3}{|c|}{ Maternal age at birth (years) $(N=1473)$} \\
\hline$<20$ & 246 & 12.4 \\
\hline $20-29$ & 845 & 56.5 \\
\hline $30-39$ & 369 & 29.7 \\
\hline$\geq 40$ & 13 & 1.4 \\
\hline \multicolumn{3}{|c|}{ Maternal Smoking during pregnancy $(N=1478)$} \\
\hline Yes & 233 & 20.0 \\
\hline No & 1245 & 80.0 \\
\hline
\end{tabular}

a $\mathrm{N}$ : unweighted $N$.

b PIR: family poverty income ratio as defined by NHANES.

The association of the various phthalate metabolites with diagnosis of ADD and/or LD is shown in Table 4. Each 10-fold increase in $\Sigma$ DEHP concentrations was associated with a 2-fold increase in odds of ADD (odds ratio $(O R)=2.1$, 95\% Confidence Interval (CI): 1.1, 3.9). Each 10-fold increase in shigh M.W. phthalate concentrations was associated with an adjusted odds ratio for ADD of 2.7 (95\% CI: 1.2, 6.1). No other urinary phthalate metabolite concentrations were associated with ADD. Sex of the child modified the association between MEP and ADD (interaction $p=0.04$ ); however, the association included 1 for both sexes (Table 5 ).

We found no significant associations of urinary phthalate metabolites with LD. Although $\Sigma$ DEHP and shigh M.W. phthalate concentrations were marginally associated with LD in unadjusted models (OR=1.6; 95\% CI: 0.9, 2.8 and OR: 2.0; 95\% CI: 1.0, 3.9, respectively), upon adjusting for potential confounders, these associations were no longer observed. We found no effect modification by sex (data not shown).

In models of co-occurring ADD and LD, the adjusted odds ratio for each 10 -fold increase in $\Sigma$ DBP and $\Sigma$ high M.W. phthalates was 3.3 (95\% CI: 0.9, 12.7) and 3.7 (95\% CI: 0.9, 14.8) (Table 4), respectively, albeit not statistically significant. Although no other metabolites were significantly related to ADD and LD in the overall models, we found significant effect modification by sex in the association of $\Sigma$ DEHP and co-occurrence of ADD and LD $(p=0.003)$. The odds ratio associated with each 10 -fold increase in $\Sigma$ DEHP levels was 6.2 for females (95\% CI: 1.7, 22.7) but 0.9 in males (95\% CI: 0.3, 2.7). There was evidence that the ORs for MCPP and both ADD and $\operatorname{LD}(p=0.05)$ differed for males and females, but confidence intervals included 1 for both sexes (Table 5).

No significant interaction was found between child's blood lead and phthalate concentrations. Models using the $\log _{10}$ of phthalate concentrations without creatinine adjustment gave similar results.

\section{Discussion}

In NHANES, a nationally representative survey of the U.S. population, we found that children's urinary concentrations of metabolites of certain phthalates, commonly used as plasticizers, were related to diagnoses of ADD in school-age children 6-15 years old. Children had twice the odds of having ADD with every 10 -fold increase in $\Sigma$ DEHP concentrations, and three times the odds of having both ADD and LD for every 10-fold increase in high M.W. phthalates. Effect modification by sex was statistically significant in some models with phthalate concentrations to be more likely associated with ADD or ADD and LD in girls than in boys; however, stratum-specific ORs usually included 1 for both sexes. Only in the association between $\Sigma$ DEHP and co-occurrence of ADD and LD was the association significant for girls. A main strength of our study is that our results are highly generalizable to the U.S. population, unlike previous studies (Engel et al., 2010, 2009; Kim et al., 2009; Whyatt et al., 2012). In addition, to the best of our knowledge, this is the first study to investigate LD as well as both ADD and LD as an outcome for phthalate exposures.

Since NHANES does not provide longitudinal data, we did not have data on in utero exposure and could not control for it in our analyses. This may be the time of greatest vulnerability. In addition, due to the cross-sectional design, we could not establish causality in the relationship between phthalates and ADD or LD and reverse causality remains a possibility. This study is also limited by the fact that phthalate exposure is estimated by metabolite concentrations in a single urine sample. Phthalate diesters are broken down into monoester metabolites in the body and excreted in urine within $24 \mathrm{~h}$, suggesting that urinary measurements represent short-term exposure (Anderson et al., 2001; CDC, 2009; Wittassek and Angerer, 2008). However, urinary metabolite concentrations have been shown to be fairly stable over six months, likely due to regular, ongoing human exposure to phthalate-containing products (Teitelbaum et al., 2008).

Additionally, our analyses are potentially limited by reliance on parent-reported ADD and LD as outcome measures rather than diagnostic data based on DSM-IV criteria in NHANES. However, it is important to note that the DSM-IV restricted data is not free of bias as it is collected by interviewers with no clinical training and relies on parent reported symptoms, ultimately sharing the same limitation of parent recall bias as the public data. Questions about current ADD or LD were not added to NHANES until 2007, which would minimize such a bias (CDC, 2010). Research suggests that parent-reported ADHD is fairly reliable and accurate, with 94\% sensitivity and 97\% specificity for maternal recall of ADHD diagnosis over a one-year period (Faraone et al., 1995). Further, it has been shown that parentreported ADHD is as sensitive as teacher-reported ADHD in a clinical trial setting (Biederman et al., 2004), with a 90\% likelihood of corroboration leading to clinical diagnosis (Biederman et al., 1990).

Another limitation of this study is that we could not include one metabolite (MECPP) in our $\Sigma$ DEHP and shigh M.W. phthalate variables, since NHANES did not measure this in 2001-2002. It has been shown that MECPP and other oxidative metabolites of DEHP are better biomarkers for exposure assessment in the US population than MEHP, a non-oxidative metabolite (Silva et al., 2006). 
Table 2

Concentrations of urinary phthalate metabolites ( $N=1493)$, NHANES (2001-2004).

\begin{tabular}{|c|c|c|c|c|c|c|c|c|c|c|}
\hline \multirow[t]{2}{*}{ Phthalate ( $\mu \mathrm{g} / \mathrm{g}$ creatinine) } & \multirow[t]{2}{*}{ LOD $^{\mathrm{a}}$} & \multirow[t]{2}{*}{$>$ LOD $(\%)$} & \multirow[t]{2}{*}{$\mathrm{GM}^{\mathrm{b}}\left(95 \% \mathrm{CI}^{\mathrm{c}}\right)$} & \multicolumn{7}{|c|}{ Percentile } \\
\hline & & & & 0 & 10 & 25 & 50 & 75 & 90 & 100 \\
\hline$\Sigma D E H P^{d}$ & & & $56.9(52.0,62.2)$ & 1.5 & 17.6 & 29.2 & 50.7 & 94.8 & 181.3 & 3613.5 \\
\hline MEHP & 0.9 & 75.4 & $3.4(3.0,3.8)$ & $<$ LOD & $<$ LOD & 1.6 & 3.5 & 7.5 & 16.4 & 294.2 \\
\hline MEHHP & 0.3 & 99.7 & $30.6(27.9,33.6)$ & $<$ LOD & 9.3 & 15.4 & 27.1 & 52.1 & 102.2 & 2062.7 \\
\hline MEOHP & 0.5 & 99.7 & $21.2(19.4,23.1)$ & $<$ LOD & 6.7 & 10.8 & 18.6 & 35.2 & 69.6 & 1301.8 \\
\hline$\Sigma$ DBP & & & $36.1(33.6,38.9)$ & 0.51 & 13.3 & 21.7 & 36.3 & 62.0 & 97.8 & 1924.7 \\
\hline MiBP & 0.3 & 99.5 & $4.6(4.1,5.2)$ & $<$ LOD & 1.5 & 2.7 & 4.9 & 8.9 & 15.3 & 140.1 \\
\hline MnBP & 0.4 & 99.9 & $30.2(28.2,32.2)$ & $<$ LOD & 10.8 & 17.6 & 30.3 & 50.9 & 84.3 & 1912.9 \\
\hline MBzP & 0.1 & 100.0 & $29.4(27.3,31.6)$ & 0.1 & 7.8 & 13.0 & 24.7 & 48.7 & 96.3 & 917.0 \\
\hline MCHP & 0.2 & 15.5 & $0.2(0.2,0.2)$ & $<$ LOD & $<$ LOD & $<$ LOD & $<$ LOD & $<$ LOD & 0.5 & 15.4 \\
\hline MEP & 0.4 & 100.0 & $114.1(104.6,124.5)$ & 2.7 & 40.9 & 65.3 & 131.8 & 296.4 & 687.8 & 13187.5 \\
\hline MiNP & 1.0 & 9.1 & $0.7(0.6,0.7)$ & $<$ LOD & $<$ LOD & $<$ LOD & $<$ LOD & $<$ LOD & $<$ LOD & 23.0 \\
\hline MMP & 1.0 & 62.4 & $1.7(1.5,2.0)$ & $<$ LOD & $<$ LOD & $<$ LOD & 1.8 & 3.6 & 7.7 & 431.8 \\
\hline$\Sigma D O P$ & & & $6.4(6.0,6.8)$ & 0.5 & 2.3 & 3.4 & 5.3 & 8.8 & 14.7 & 77.8 \\
\hline МСРР & 0.2 & 99.9 & $5.3(5.0,5.7)$ & $<$ LOD & 1.7 & 2.7 & 4.5 & 7.7 & 13.7 & 76.4 \\
\hline MOP & 1.0 & 8.4 & $0.7(0.7,0.8)$ & $<$ LOD & $<$ LOD & $<$ LOD & $<$ LOD & $<$ LOD & 1.6 & 14.0 \\
\hline$\Sigma$ High M.W. $^{\mathrm{e}}$ & & & $105.0(98.9,111.6)$ & 3.3 & 35.3 & 55.2 & 92.3 & 161.6 & 292.3 & 3741.3 \\
\hline$\Sigma$ Low M.W. & & & $177.0(163.4,191.8)$ & 10.8 & 73.9 & 108.7 & 191.5 & 371.4 & 756.5 & 13262.4 \\
\hline
\end{tabular}

a LOD: limit of detection.

b GM: geometric mean.

c CI: confidence interval.

d Sum of DEHP does not include MECPP because it was measured only in NHANES (2003-2004).

e $\Sigma$ High M.W.: sum of high molecular weight phthalates $=\mathrm{MBzP}+\mathrm{MCPP}+\Sigma \mathrm{DEHP}[\mathrm{MEHP}+\mathrm{MEHHP}+\mathrm{MEOHP}]$.

${ }^{\mathrm{f}} \Sigma$ Low M.W.: sum of low molecular weight phthalates $=\mathrm{MEP}+\mathrm{MMP}+\Sigma \mathrm{DBP}[\mathrm{MiBP}+\mathrm{MnBP}]$.

Table 3

Geometric means of urinary phthalate metabolites ( $\mu \mathrm{g} / \mathrm{g}$ creatinine) by diagnosis in study population $(N=1490)$.

\begin{tabular}{|c|c|c|c|c|c|}
\hline & $\begin{array}{l}\text { Neither }(N=1262) \\
\text { GM }(95 \% \mathrm{CI})\end{array}$ & $\begin{array}{l}\text { ADD only }(N=56) \\
\text { GM }(95 \% \mathrm{CI})\end{array}$ & $\begin{array}{l}\text { LD only }{ }^{\mathrm{b}}(N=116) \\
\text { GM }(95 \% \mathrm{CI})\end{array}$ & $\begin{array}{l}\text { ADD + LD }(N=56) \\
\text { GM }(95 \% \mathrm{CI})\end{array}$ & $p$ Value $^{c}$ \\
\hline$\Sigma$ DEHP & $55.1(50.3,60.4)$ & $66.8(52.7,84.7)$ & $58.6(45.6,75.4)$ & $81.0(55.6,118.0)$ & 0.04 \\
\hline$\Sigma \mathrm{DBP}$ & $35.9(33.4,38.6)$ & $31.7(24.3,41.3)$ & $33.3(27.5,40.5)$ & $49.3(36.4,66.8)$ & 0.28 \\
\hline MBzP & $28.7(26.6,31.0)$ & $25.8(17.6,38.0)$ & $28.8(22.3,37.3)$ & $46.6(29.0,75.1)$ & 0.14 \\
\hline MEP & $116.4(105.4,128.5)$ & $90.5(61.4,133.4)$ & $106.1(91.6,123.0)$ & $108.5(71.9,164.0)$ & 0.45 \\
\hline MCPP & $5.3(4.9,5.7)$ & $5.4(4.0,7.4)$ & $5.2(4.4,6.3)$ & $6.2(4.9,7.7)$ & 0.36 \\
\hline$\Sigma$ High M.W. & $101.6(95.6,107.9)$ & $111.7(87.5,142.6)$ & $105.1(84.4,131.0)$ & $171.8(116.8,252.7)$ & 0.02 \\
\hline ¿Low M.W. & $179.5(163.6,196.9)$ & $141.4(102.5,195.0)$ & $157.6(138.3,179.7)$ & $193.6(137.2,273.1)$ & 0.75 \\
\hline
\end{tabular}

a ADD only: children with a diagnosis of ADD but not LD.

${ }^{\mathrm{b}}$ LD only: children with a diagnosis of LD but not ADD.

c Test of trend $p$-value.

Table 4

Odds ratios for ADD $(N=1491)$, LD $(N=1493)$, and ADD + LD $(N=1318)$ for 10 -fold increases in urinary phthalate metabolite levels.

\begin{tabular}{|c|c|c|c|c|c|c|}
\hline & \multicolumn{2}{|l|}{$\operatorname{ADD}(N=112)$} & \multicolumn{2}{|l|}{$\operatorname{LD}(N=173)$} & \multicolumn{2}{|l|}{$\mathrm{ADD}+\mathrm{LD}(N=56)$} \\
\hline & Unadjusted OR ${ }^{\mathrm{a}}(95 \% \mathrm{CI})$ & Adjusted $^{\mathrm{b}} \mathrm{OR}^{\mathrm{a}}(95 \% \mathrm{CI})$ & Unadjusted OR ${ }^{\mathrm{a}}(95 \% \mathrm{CI})$ & Adjusted $^{\mathrm{b}} \mathrm{OR}^{\mathrm{a}}(95 \% \mathrm{CI})$ & Unadjusted OR ${ }^{\mathrm{a}}(95 \% \mathrm{CI})$ & Adjusted $^{\mathrm{b}} \mathrm{OR}^{\mathrm{a}}(95 \% \mathrm{CI})$ \\
\hline$\Sigma$ DEHP & $2.0(1.2,2.3)^{*}$ & $2.1(1.1,3.9)^{*}$ & $1.6(0.9,2.8)^{* * * *}$ & $1.5(0.7,3.0)$ & $2.4(1.1,5.3)^{*}$ & $2.2(0.6,7.4)$ \\
\hline$\Sigma \mathrm{DBP}$ & $1.5(0.8,2.9)$ & $1.8(0.6,4.8)$ & $1.4(0.7,2.7)$ & $1.3(0.6,2.9)$ & $2.5(1.1,5.6)^{*}$ & $3.3(0.9,12.7)^{\text {***** }}$ \\
\hline MBzP & $1.7(0.9,3.2)$ & $1.5(0.7,3.4)$ & $1.6(0.8,3.0)$ & $1.2(0.6,2.5)$ & $2.7(1.1,6.4)^{*}$ & $2.0(0.6,6.3)$ \\
\hline MEP & $0.8(0.4,1.4)$ & $1.0(0.6,1.8)$ & $0.9(0.6,1.4)$ & $1.0(0.6,1.6)$ & $0.9(0.4,2.0)$ & $1.7(0.9,3.3)$ \\
\hline МСРР & $1.4(0.7,2.8)$ & $1.2(0.4,4.0)$ & $1.2(0.7,2.1)$ & $0.9(0.4,1.9)$ & $1.7(0.8,3.8)$ & $1.0(0.2,4.7)$ \\
\hline इHigh M.W. & $2.7(1.4,5.1)^{* * *}$ & $2.7(1.2,6.1)^{*}$ & $2.0(1.0,3.9)^{*}$ & $1.6(0.7,3.6)$ & $4.3(1.6,11.8)^{* * * k}$ & $3.7(0.9,14.8)^{* * * * *}$ \\
\hline ¿Low M.W. & $0.9(0.4,1.9)$ & $1.1(0.5,2.4)$ & $0.9(0.5,1.7)$ & $1.0(0.5,1.9)$ & $1.2(0.5,3.1)$ & $2.2(0.8,6.2)$ \\
\hline
\end{tabular}

$* p<0.05$.

*** $p<0.01$.

**** $p<0.10$.

a OR: odds ratio.

${ }^{\mathrm{b}}$ Adjusted for child sex, age, race, household income, blood lead, and maternal smoking during pregnancy.

Our results corroborate some of the findings from previous studies. The only other cross-sectional study to date found that DEHP, but not DBP, was associated with increased odds of ADHD in
Korean children (Kim et al., 2009). We confirmed these results with ADD as well as co-occurring ADD and LD. Although the Korean study did not examine effect modification by sex, a study 
Table 5

Odds ratios for $\mathrm{ADD}$ and $\mathrm{ADD}+\mathrm{LD}$ for 10 -fold increases in urinary phthalate metabolite levels, stratified by sex.

\begin{tabular}{|c|c|c|c|c|c|c|}
\hline & \multicolumn{3}{|l|}{ ADD } & \multicolumn{3}{|l|}{$\mathrm{ADD}+\mathrm{LD}$} \\
\hline & $\begin{array}{l}\text { Male OR }{ }^{\mathrm{a}}(95 \% \mathrm{CI}) \\
(N=726)\end{array}$ & $\begin{array}{l}\text { Female } \mathrm{OR}^{\mathrm{a}}(95 \% \mathrm{CI}) \\
(N=765)\end{array}$ & $\begin{array}{l}\text { Interaction term } \\
p \text { value }(N=1491)\end{array}$ & $\begin{array}{l}\text { Male } \mathrm{OR}^{\mathrm{a}}(95 \% \mathrm{CI}) \\
(N=613)\end{array}$ & $\begin{array}{l}\text { Female } \mathrm{OR}^{\mathrm{a}}(95 \% \mathrm{CI}) \\
(N=705)\end{array}$ & $\begin{array}{l}\text { Interaction term } \\
p \text { value }(N=1318)\end{array}$ \\
\hline$\Sigma$ DEHP & $1.5(0.7,3.1)$ & $3.5(1.0,11.8)^{*}$ & 0.26 & $0.9(0.3,2.7)$ & $6.2(1.7,22.7)^{* * *}$ & 0.003 \\
\hline$\Sigma \mathrm{DBP}$ & $1.7(0.5,5.4)$ & $2.0(0.4,8.8)$ & 0.85 & $2.1(0.3,17.0)$ & $6.4(1.4,28.7)^{*}$ & 0.36 \\
\hline MBzP & $1.4(0.5,3.6)$ & $2.1(0.8,5.5)$ & 0.48 & $1.9(0.5,7.1)$ & $2.5(0.7,9.6)$ & 0.68 \\
\hline MEP & $1.3(0.7,2.4)$ & $0.5(0.2,1.1)^{* * * *}$ & 0.04 & $2.2(0.8,5.7)$ & $0.8(0.3,1.9)$ & 0.21 \\
\hline МСРP & $1.1(0.3,4.3)$ & $1.7(0.5,5.8)$ & 0.53 & $0.6(0.1,3.8)$ & $2.8(0.6,13.5)$ & 0.05 \\
\hline ¿High M.W. & $2.1(0.8,6.1)$ & $4.1(0.9,19.3)^{* * * * *}$ & 0.51 & $2.1(0.3,12.9)$ & $8.6(1.7,43.1)^{*}$ & 0.20 \\
\hline sLow M.W. & $1.4(0.6,3.2)$ & $0.7(0.2,2.0)$ & 0.26 & $2.4(0.6,10.2)$ & $1.7(0.5,5.3)$ & 0.73 \\
\hline
\end{tabular}

investigating prenatal exposure to phthalates confirmed our report that higher concentrations of some high M.W. phthalate metabolites (including DEHP) are associated with decreased attention and alertness primarily in females (Engel et al., 2009). However, in contrast to our findings, a cross sectional study found a negative association between DEHP metabolites and IQ scores among boys but not girls (Cho et al., 2010).

In part, the stronger association between some phthalates and ADD and LD in females than males may derive from the higher likelihood that they use phthalate-containing personal care products, such as body lotions, perfumes, and deodorants (RomeroFranco et al., 2011). For example, in NHANES, girls had significantly higher urinary concentrations of MEP, $\Sigma$ DBP, and $\Sigma$ low M.W. phthalates than boys. However, females did not have higher levels of $\Sigma$ high M.W. phthalates including MBzP, MCPP and $\Sigma$ DEHP. Thus, this hypothesis cannot explain why $\Sigma$ DEHP were associated with ADD and LD in girls but not boys.

Biological mechanisms underlying the association between certain phthalates and childhood developmental disorders are uncertain. Peroxisome proliferator-activated receptors (PPARs) have been detected in neural tubes of rat embryos (Braissant and Wahli, 1998) and phthalates have been shown to activate these receptors (Roberts et al., 1997). PPAR signal transduction pathways have been linked to neurodegenerative diseases and cognitive functioning by mechanisms of inflammation and lipid metabolism (van Neerven et al., 2008). In utero exposure to DEHP has been shown to decrease lipid concentration and composition in the fetal rat brain (Innis, 2003; Xu et al., 2007).

An alternative biological explanation is that phthalates impact brain development through their effects on thyroid function (Hinton et al., 1986; Price et al., 1988). Thyroid hormones play a key role in neurodevelopment and hippocampal function, and delayed or impaired brain differentiation and hippocampal dysfunction often result in deficits in learning and memory in rats such as impaired spatial maze memory (Akaike et al., 1991; Nunez, 1984; Porterfield and Hendry, 1998; Stein et al., 1991; Vaccari, 1988). Exposure to low levels of phthalates can result in loss of midbrain dopaminergic nuclei (Tanida et al., 2009). Research suggests that aberrations in the dopamine system play a major role in the pathophysiology of ADD and other neuropsychiatric disorders (Krause, 2008; Krause et al., 2003; Shen et al., 2012; Spencer et al., 2005).

\section{Conclusion}

Our study using representative data from the general U.S. population adds to the body of evidence suggesting an association between phthalate exposure and childhood developmental disorders, specifically ADD and LD. We found that $\mathrm{DDEHP}$ and $\Sigma$ high M.W. phthalates were associated with increased odds for ADD as well as both ADD and LD, especially in girls.

\section{Funding source}

This work was supported by Grants PO1 ES009605 from the National Institute of Environmental Health Sciences and RD83451301 from the United States Environmental Protection Agency.

\section{Disclaimer}

The findings and conclusions in this report are those of the authors and do not necessarily represent the views of the funders.

\section{Financial disclosure}

The authors have no financial conflict of interest to disclose.

\section{IRB approval}

The Committee for Protection of Human Subjects (CPHS) at UC Berkeley determined that our research did not meet the criteria for human research as per federal regulations, and therefore did not require review.

\section{References}

Afshari, A., et al., 2004. Emission of phthalates from PVC and other materials Indoor Air 14, 120-128.

Akaike, M., et al., 1991. Hyperactivity and spatial maze learning impairment of adult rats with temporary neonatal hypothyroidism. Neurotoxicol. Teratol. 13, 317-322.

Anderson, W.A., et al., 2001. A biomarker approach to measuring human dietary exposure to certain phthalate diesters. Food Addit. Contam. 18, 1068-1074.

APA, 1994. Diagnostic and Statistical Manual of Mental Disorders. American Psychiatric Association, Washington, DC.

Arcadi, F.A., et al., 1998. Oral toxicity of bis(2-ethylhexyl) phthalate during pregnancy and suckling in the Long-Evans rat. Food Chem. Toxicol. 36, 963-970.

Barr, D.B., et al., 2003. Assessing human exposure to phthalates using monoesters and their oxidized metabolites as biomarkers. Environ. Health Perspect. 111, 1148-1151.

Biederman, J., et al., 2004. How informative are parent reports of attention-deficit/ hyperactivity disorder symptoms for assessing outcome in clinical trials of long-acting treatments? A pooled analysis of parents' and teachers' reports. Pediatrics 113, 1667-1671. 
Biederman, J., et al., 1990. Parent-based diagnosis of attention deficit disorder predicts a diagnosis based on teacher report. J. Am. Acad. Child. Adolesc. Psychiatry 29, 698-701.

Boas, M., et al., 2010. Childhood exposure to phthalates: associations with thyroid function, insulin-like growth factor I, and growth. Environ. Health Perspect. 118, $1458-1464$.

Braissant, O., Wahli, W., 1998. Differential expression of peroxisome proliferatoractivated receptor-alpha, -beta, and -gamma during rat embryonic development. Endocrinology 139, 2748-2754.

CDC, 2012. About the National Health and Nutrition Examination Survey (vol.).

CDC, 2006. Phthalate Metabolites in Urine: NHANES 2003-2004. In: E. Health (Ed.)

CDC, 2009. Fourth National Report on Human Exposure to Environmental Chemicals. 〈http://www.cdc.gov/exposurereport/〉.

CDC, 2010. Increasing Prevalence of Parent-Reported Attention-Deficit/Hyperactivity Disorder Among Children-United States, 2003 and 2007. 〈http://www.cdc. gov/mmwr/preview/mmwrhtml/mm5944a3.htm $\rangle$.

CDC, 2011. Continuous NHANES Web Tutorial. 〈http://www.cdc.gov/nchs/tutorials/ NHANES/SurveyDesign/intro.htm>.

Chen, T., et al., 2011. Mono-(2-ethylhexyl) phthalate impairs neurodevelopment: inhibition of proliferation and promotion of differentiation in PC12 cells. Toxicol. Lett. 201, 34-41.

Cho, S.C., et al., 2010. Relationship between environmental phthalate exposure and the intelligence of school-age children. Environ. Health Perspect. 118, 1027-1032.

Clark, K., et al., 2003. Assessment of critical exposure pathways. Part Q: phthalate esters. In: CA, S. (Ed.), The Handbook of Environmental Chemistry. Springer, New York.

Clausen, P.A., et al., 2004. Emission of di-2-ethylhexyl phthalate from PVC flooring into air and uptake in dust: emission and sorption experiments in FLEC and CLIMPAQ. Environ. Sci. Technol. 38, 2531-2537.

Colborn, T., 2004. Neurodevelopment and endocrine disruption. Environ. Health Perspect. 112, 944-949.

Crofton, K.M., 2008. Thyroid disrupting chemicals: mechanisms and mixtures. Int. J. Androl. 31, 209-223.

Engel, S.M., et al., 2010. Prenatal phthalate exposure is associated with childhood behavior and executive functioning. Environ. Health Perspect. 118, 565-571.

Engel, S.M., et al., 2009. Prenatal phthalate exposure and performance on the Neonatal Behavioral Assessment Scale in a multiethnic birth cohort. Neurotoxicology 30, 522-528.

Faraone, S.V., et al., 1995. How reliable are maternal reports of their children's psychopathology? One-year recall of psychiatric diagnoses of ADHD children. J. Am. Acad. Child. Adolesc. Psychiatry 34, 1001-1008.

Hauser, R., Calafat, A.M., 2005. Phthalates and human health. Occup. Environ. Med. $62,806-818$

Hinton, R.H., et al., 1986. Effects of phthalic acid esters on the liver and thyroid Environ. Health Perspect. 70, 195-210.

Hornung, R.W., Reed, L.D., 1990. Estimation of average concentration in the presence of nondetectable values. Appl. Occup. Environ. Hyg. 5, 46-51.

Innis, S.M., 2003. Perinatal biochemistry and physiology of long-chain polyunsaturated fatty acids. J. Pediatr. 143, S1-S8.

Ishido, M., et al., 2004. Dicyclohexylphthalate causes hyperactivity in the rat concomitantly with impairment of tyrosine hydroxylase immunoreactivity. J. Neurochem. 91, 69-76.

Jackson, S., 1966. Creatinine in urine as an index of urinary excretion rate. Health Phys. 12, 843-850.

Kavlock, R.J., et al., 1996. Research needs for the risk assessment of health and environmental effects of endocrine disruptors: a report of the U.S. EPAsponsored workshop. Environ. Health Perspect. 104 (Suppl. 4), S715-S740.

Kim, B.N., et al., 2009. Phthalates exposure and attention-deficit/hyperactivity disorder in school-age children. Biol. Psychiatry 66, 958-963.

Krause, J., 2008. SPECT and PET of the dopamine transporter in attention-deficit/ hyperactivity disorder. Expert Rev. Neurother. 8, 611-625.

Krause, K.H., et al., 2003. The dopamine transporter and neuroimaging in attention deficit hyperactivity disorder. Neurosci. Biobehav. Rev. 27, 605-613.

Li, Y., et al., 2009. Neurobehavioral toxicity study of dibutyl phthalate on rats following in utero and lactational exposure. J. Appl. Toxicol. 29, 603-611.
Masuo, Y., et al., 2004. Motor hyperactivity caused by a deficit in dopaminergic neurons and the effects of endocrine disruptors: a study inspired by the physiological roles of PACAP in the brain. Regul. Pept. 123, 225-234.

Mattison, R.E., Mayes, S.D., 2012. Relationships between learning disability, executive function, and psychopathology in children with ADHD. J. Atten. Disord. 16, $138-146$.

NHANES, 2004. Laboratory Procedures Manual.

NRC, 2008. National Research Council. Committee on the Health Risks of Phthalates. Phthalates and Cumulative Risk Asssessment: The Task Ahead. National Academy Press, Washington, DC.

Nunez, J., 1984. Effects of thyroid hormones during brain differentiation. Mol. Cell Endocrinol. 37, 125-132.

Porterfield, S.P., Hendry, L.B., 1998. Impact of PCBs on thyroid hormone directed brain development. Toxicol. Ind. Health 14, 103-120.

Price, S.C., et al., 1988. Alterations in the thyroids of rats treated for long periods with di-(2-ethylhexyl) phthalate or with hypolipidaemic agents. Toxicol. Lett. $40,37-46$.

Roberts, R.A., et al., 1997. Perturbation of the mitosis/apoptosis balance: a fundamental mechanism in toxicology. Fundam. Appl. Toxicol. 38, 107-115.

Romero-Franco, M., et al., 2011. Personal care product use and urinary levels of phthalate metabolites in Mexican women. Environ. Int. 37, 867-871.

Sathyanarayana, S., et al., 2008. Baby care products: possible sources of infant phthalate exposure. Pediatrics 121, e260-e268.

Shen, L.H., et al., 2012. Recent advances in imaging of dopaminergic neurons for evaluation of neuropsychiatric disorders. J. Biomed. Biotechnol. 2012, 259349.

Silva, M.J., et al., 2004. Urinary levels of seven phthalate metabolites in the U.S. population from the National Health and Nutrition Examination Survey (NHANES) 1999-2000. Environ. Health Perspect. 112, 331-338.

Silva, M.J., et al., 2006. Measurement of eight urinary metabolites of $\operatorname{di}(2-$ ethylhexyl) phthalate as biomarkers for human exposure assessment. Biomarkers $11,1-13$.

Smith, C.A., et al., 2011. Acute postnatal exposure to di(2-ethylhexyl) phthalate adversely impacts hippocampal development in the male rat. Neuroscience 193, 100-108.

Spencer, T.J., et al., 2005. In vivo neuroreceptor imaging in attention-deficit/ hyperactivity disorder: a focus on the dopamine transporter. Biol. Psychiatry 57, 1293-1300.

Stein, S.A., et al., 1991. Thyroid hormone control of brain and motor development: molecular, neuroanatomical, and behavioral studies. Adv. Exp. Med. Biol. 299, 47-105.

Tanaka, T., 2005. Reproductive and neurobehavioural effects of bis(2-ethylhexyl) phthalate (DEHP) in a cross-mating toxicity study of mice. Food Chem. Toxicol. $43,581-589$.

Tanida, T. et al., 2009. Fetal and neonatal exposure to three typical environmental chemicals with different mechanisms of action: mixed exposure to phenol, phthalate, and dioxin cancels the effects of sole exposure on mouse midbrain dopaminergic nuclei. Toxicol. Lett. 189, 40-47.

Teitelbaum, S.L., et al., 2008. Temporal variability in urinary concentrations of phthalate metabolites, phytoestrogens and phenols among minority children in the United States. Environ. Res. 106, 257-269.

Vaccari, A., 1988. Teratogenic mechanisms of dysthyroidism in the central nervous system. Prog. Brain Res. 73, 71-86.

Vakil, E., et al., 2012. Verbal learning and memory as measured by the Rey-Auditory Verbal Learning Test: ADHD with and without learning disabilities. Child Neuropsychol. 18, 449-466.

van Neerven, S., et al., 2008. RAR/RXR and PPAR/RXR signaling in neurological and psychiatric diseases. Prog. Neurobiol. 85, 433-451.

Whyatt, R.M., et al., 2012. Maternal prenatal urinary phthalate metabolite concentrations and child mental, psychomotor, and behavioral development at 3 years of age. Environ. Health Perspect. 120, 290-295.

Wittassek, M., Angerer, J., 2008. Phthalates: metabolism and exposure. Int. J. Androl. 31, 131-138.

Wolff, M.S., et al., 2008. Prenatal phenol and phthalate exposures and birth outcomes. Environ. Health Perspect. 116, 1092-1097.

Xu, Y., et al., 2007. Di-(2-ethylhexyl)-phthalate affects lipid profiling in fetal rat brain upon maternal exposure. Arch. Toxicol. 81, 57-62. 